\title{
Intrahepatic Cholangiocarcinoma and Thermal Ablation: Long-term Results of An Italian Retrospective Multicenter Study
}

\author{
Antonio Giorgio*1, Pietro Gatti ${ }^{2}$, Luca Montesarchio ${ }^{3}$, Bruno Santoro ${ }^{1}$, Andrea Dell'Olio ${ }^{4}$, \\ Nicola Crucinio ${ }^{5}$, Carmine Coppola ${ }^{6}$, Ferdinando Scarano ${ }^{6}$, Fabio De Biase ${ }^{5}$, Emanuela Ciraci ${ }^{7}$, \\ Stefano Semeraro ${ }^{7}$ and Valentina Giorgio ${ }^{8}$ \\ ${ }^{1}$ Liver Unit and Interventional Ultrasound Unit, Athena Clinical Institute, Piedimonte Matese (CE), Italy; ${ }^{2}$ Internal Medicine Unit, \\ Brindisi General Hospital, Brindisi, Italy; ${ }^{3}$ General Surgery Unit, Polla General Hospital, Polla (SA), Italy; ${ }^{4}$ Department of \\ Radiology, Bisceglie Hospital - ASL BAT, Bisceglie, Italy; ${ }^{5}$ Gastroenterology Unit, Foggia General Hospital, Foggia, Italy; \\ ${ }^{6}$ Department of Internal Medicine, Hepatology Interventional Unit, Gragnano Hospital, Gragnano (NA), Italy; ${ }^{7}$ Internal Medicine \\ Unit, Ostuni Hospital, Ostuni (BR), Italy; ${ }^{8}$ Fondazione Policlinico A. Gemelli IRCCS, Department of Woman and Child Health and \\ Public Health, Largo A Gemelli, Roma, Italy
}

\begin{abstract}
Background and Aims: Despite resection being considered the treatment of choice for intrahepatic cholangiocarcinoma (ICC), percutaneous thermal ablation can be an alternative treatment for patients unfit for surgery. Our aim was to compare long-term results of percutaneous sonographically-guided radiofrequency ablation (RFA) with highpowered microwave ablation (MWSA) in treatment of ICC. Methods: Results of 71 ICC patients with 98 nodules treated with RFA (36 patients) or MWSA (35 patients) between January 2008 and June 2018 in 5 Interventional Ultrasound centers of Southern Italy were retrospectively reviewed. Cumulative overall survival curves were calculated with the Kaplan-Meyer method and differences with the log-rank test. Eleven possible factors affecting survival were analyzed. Results: Overall survival of the entire series was $88 \%$, $65 \%, 45 \%$ and $34 \%$ at $12,36,60$ and 80 months, respectively. Patients treated with MWSA survived longer than patients treated with RFA $(p<0.005)$. The MWSA group with ICC nodules $\leq 3 \mathrm{~cm}$ or nodules up to $4 \mathrm{~cm}$ survived longer than the RFA group $(p<0.0005)$. In patients with nodules $>4 \mathrm{~cm}$, no significant difference was found. Disease-free survival and progression-free survival were better in the MWSA group compared to the RFA group $(p<0.005)$. Diameter of nodules and MWSA were independent factors predicting a better survival. No major complications were observed. Conclusions: MWSA is superior to RFA in treating ICC unfit for surgery, achieving better long-term survival in small $(\leq 3 \mathrm{~cm})$ ICC nodules as well as nodules up to $4 \mathrm{~cm}$ of neoplastic tumors and should replace RFA.
\end{abstract}

Keywords: Intrahepatic cholangiocarcinoma; Thermal ablation; Microwaves; Radiofrequency; Percutaneous treatment.

Abbreviations: CECT, contrast-enhanced computed tomography; CEMRI, contrast-enhanced magnetic resonance imaging; CEUS, contrast-enhanced ultrasound; ICC, cholangiocarcinoma; MWSA, microwaves ablation; OS, overall survival; RFA, radiofrequency ablation; US, ultrasound.

Received: 14 August 2019; Revised: 15 October 2019; Accepted: 6 November 2019

*Correspondence to: Antonio Giorgio, Liver Unit and Interventional Ultrasound Unit, Athena Clinical Institute, Piedimonte Matese (CE) (81016), Italy. Tel: +39823784666, E-mail: agiorgio28@gmail.com
Citation of this article: Giorgio $A$, Gatti $P$, Montesarchio $L$, Santoro B, Dell'Olio A, Crucinio N, et al. Intrahepatic cholangiocarcinoma and thermal ablation: long-term results of an Italian retrospective multicenter study. J Clin Transl Hepatol 2019;7(4):287-292. doi: 10.14218/JCTH.2019.00036.

\section{Introduction}

Intrahepatic cholangiocarcinoma (ICC) is the second most common primary malignant tumor of the liver. ${ }^{1,2}$ ICC accounts for up to $8-10 \%$ of all cholangiocarcinomas and $10-20 \%$ of all primary liver tumors. ${ }^{1}$ Mortality from ICC has risen globally, ${ }^{2}$ and recent data have indicated that the occurrence of ICC on cirrhosis is increasing. ${ }^{1,2}$ ICC is considered more aggressive than hepatocellular carcinoma. Resection is the treatment of choice because it increases survival. ${ }^{3}$ Nevertheless, some researchers have recently reported on ICC patients unfit for surgery who were treated with percutaneous thermal ablation, initially radiofrequency and later microwaves. ${ }^{4-10}$

According to the literature, survival of patients with small $(<3 \mathrm{~cm})$ tumors was better than that of patients with larger tumors. In 2017, Shindoh ${ }^{11}$ reviewed published studies on ablative therapies of ICC, which at that time were limited in number and with small numbers of treated patients. On the basis of literature data and upon the experience of his Hospital Institution, this author proposed an algorithm that considered ablation as an alternative treatment option to surgery in unresectable ICC patients. In this algorithm, radiofrequency ablation (RFA) appeared to be the technique of choice. ${ }^{11} \mathrm{We}$ also commented ${ }^{12}$ on the proposal of Shindoh and agreed that "only limited evidence from a small number of studies has been reported because of the rarity of this tumor", and that thermal "ablation therapies could be the treatment of choice for selected cases of ICC".

Nevertheless, we concluded that "most likely RFA should be replaced by microwave ablation (MWSA) that is able to ensure a complete ablation of tumor nodules $<3-3.5 \mathrm{~cm}$, determining a sufficient ablative margin of the tumor just for the properties inherent in the technique". ${ }^{12}$ In fact, compared 
to RFA, MWSA induces a larger volume of necrosis in a faster time and without the so-called sink effect. Furthermore, compared to RFA, MWSA is able to determine a spherical size of the ablated area with a predictable volume of necrosis. ${ }^{13,14}$

Therefore, the aim of the present study was to report longterm survivals of ICC unresectable patients treated with RFA compared to those treated with MWSA.

\section{Methods}

Long-term results of 71 ICC patients with 98 nodules consecutively treated with RFA or MWSA between January 2008 and June 2018 in five ultrasound (US) Interventional Units of Southern Italy were retrospectively reviewed. There were 37 males and 34 females (mean age: $74 \pm 9$ years). Diagnosis of ICC was obtained with percutaneous US-guided biopsy using a $18 \mathrm{G}$ cutting needle, in all cases. Patients were included in the study when they met the following criteria: age $>18$ years; no resectability, according to the judgement of an expert hepatobiliary surgeon of every Institution $(n=63)$; refusal of surgery $(n=8)$; absence of distant metastases; absence of vascular invasion; and absence of dilatation of the biliary tree on US, contrast-enhanced computed tomography (CECT) and/or contrast-enhanced magnetic resonance imaging (CEMRI). Presence of mild ascites was not an absolute contraindication for ablation. Patients were excluded when they had more than three nodules (with the largest one $>5 \mathrm{~cm}$ ) or a single nodule $>8 \mathrm{~cm}$. Patients with cirrhosis and superimposed ICC with a total bilirubin level $>2.5 \mathrm{mg} / \mathrm{dL}$ were also excluded. ${ }^{13,16}$

Before ablation, all patients underwent clinical and biochemical exams (including assays for Ca 19.9 and alphafetoprotein), abdominal conventional US, contrast-enhanced US (CEUS), CECT and/or CEMRI. Enhancement characteristics
Giorgio A. et al: Ablation of intrahepatic cholangiocarcinoma

were classified into four patterns: peripheral irregular rim-like hyperenhancement (type I); diffuse heterogeneous hyperenhancement (type II); diffuse homogeneous hyperenhancement (type III); and diffuse heterogeneous hypoenhancement (type IV). ${ }^{15}$ All tumors were of the mass-forming type. Tables 1-3 report the clinical, biochemical and radiological characteristics of our series according to ablation treatment. Technical effectiveness (i.e. complete ablation of the macroscopic tumor, including a margin of $0.5-1 \mathrm{~cm}$ ) was evaluated by CEUS and CECT and/or CEMRI at 1 month from the last course of a defined ablation protocol. Complete ablation of the tumor was achieved when no enhancement was shown at 1 month imaging follow-up, whereas any kind of enhancement at this stage was indicative of partial ablation. In these cases, patients were re-treated using the same thermal technique previously used. Patients were followed-up every 2 months with clinical, biochemical examinations, abdominal US, and CEUS. Follow-up CECT or CEMRI was performed every 6 months.

All ablation procedures were performed percutaneously under US guidance and under unconscious sedation by five operators (AG, PG, AD, NC, CC) who at the start of the study had at least 5 years of experience in interventional US (with a range of 5-23 years). ${ }^{13-16}$ RFA was performed using single perfused electrode needles (HITT Berthold, Erlangen, Germany [AG] and RF Medical Company, Seoul, Republic of Korea [AG and PG]) and single cold water-perfused electrode needles (Covidien Italia Spa, Segrate, Milan, Italy [PG, AD, NC and $\mathrm{CC}]$ ). MWSA was performed using a high-powered MWS device (Acculis MTA System, Medical Ltd, Hampshire, UK [AG and PG], and Amica, HS Hospital Services s.p.a, Rome, Italy $[A G, P G, A D$ and $N C]$ ) with a single antenna operating at $2450 \mathrm{MHz}$ connected with a powerful generator working from 100 to $140 \mathrm{~W}$.

Table 1. Baseline characteristics and laboratory findings of patients treated with MWSA and patients treated with RFA

\begin{tabular}{lll}
\hline Patients' characteristics & MWSA group, $n=35$ & RFA group, $n=36$ \\
\hline Age in years, mean \pm SD & $72 \pm 10$ & 75 \\
Male (\%) & $18(51.4 \%)$ & $19(52.8 \%)$ \\
Comorbidities (\%) & & $8(22.2 \%)$ \\
$\quad$ None & $6(17.1 \%)$ & $11(30.5 \%)$ \\
Obesity & $13(37.1 \%)$ & $15(41.7 \%)$ \\
HCV infection & $13(37.1 \%)$ & $2(5.6 \%)$ \\
HBV infection & $3(8.6 \%)$ & $8(22.2 \%)$ \\
Child A cirrhosis & $7(20 \%)$ & $1(2.8 \%)$ \\
Child B7 cirrhosis & $1(2.8 \%)$ & $32(88.9 \%)$ \\
Treatment history (\%) & & $4(11.1 \%)$ \\
Primary treatment & $30(85.7 \%)$ & $98500(56000-176500)$ \\
Post-surgical recurrence & $5(14.3 \%)$ & $1.2(0.9-1.70)$ \\
Laboratory findings (range) & & $57(15-245)$ \\
Platelet count in cells/dL, median & $97500(52000-178000)$ & $23(10-56)$ \\
INR, median & $1.1(0.9-1.68)$ & \\
Ca 19.9 in IU/mL, median & $53(16-250)$ & \\
AFP in mg/dL, median & $21(8-54)$ & \\
\hline
\end{tabular}

Abbreviations: AFP, alpha-fetoprotein; HCV, hepatitis B virus; HCV, hepatitis C virus; INR, international normalized ratio; MWSA, microwave ablation; RFA, radiofrequency ablation; SD, standard deviation. 
Giorgio A. et al: Ablation of intrahepatic cholangiocarcinoma

Table 2. The enhancement pattern of the intrahepatic cholangiocarcinoma treated with MWSA during the arterial phase on CEUS and CECT/CEMR, and according to lesion size

\begin{tabular}{|c|c|c|c|c|c|}
\hline \multirow[b]{2}{*}{ CECT or CEMRI } & \multicolumn{5}{|l|}{ CEUS (\%) } \\
\hline & Type I & Type II & Type III & Type IV & Total \\
\hline Type I & $29(58)$ & $1(2)$ & $0(0)$ & $0(0)$ & $30(60)$ \\
\hline Type II & $0(0)$ & $14(28)$ & $0(0)$ & $2(4)$ & $16(32)$ \\
\hline Type III & $2(4)$ & $0(0)$ & $2(4)$ & $0(0)$ & $4(8)$ \\
\hline Type IV & $0(0)$ & $0(0)$ & $0(0)$ & $0(0)$ & $0(0)$ \\
\hline Total & $31(62)$ & $15(30)$ & $2(4)$ & $2(4)$ & 50 \\
\hline \multicolumn{6}{|c|}{ Size, median (range): $3.6 \mathrm{~cm}(2.2-7.2 \mathrm{~cm})$} \\
\hline$\leq 3.0 \mathrm{~cm}$ & $5(10)$ & $0(0)$ & $2(4)$ & $0(0)$ & $7(14)$ \\
\hline $3.1-5.0 \mathrm{~cm}$ & $19(38)$ & $9(18)$ & $0(0)$ & $0(0)$ & $28(56)$ \\
\hline$>5 \mathrm{~cm}$ & $7(14)$ & $6(12)$ & $0(0)$ & $2(4)$ & $15(30)$ \\
\hline Total & $31(62)$ & $15(30)$ & $2(4)$ & $2(4)$ & 50 \\
\hline
\end{tabular}

Type I (peripheral irregular rim-like hyperenhancement); Type II (diffuse heterogeneous hyperenhancement); Type III (diffuse homogeneous hyperenhancement); Type IV (diffuse heterogeneous hypoenhancement).

Abbreviations: CEMRI, contrast-enhanced magnetic resonance imaging; CETC, contrast-enhanced computed tomography; CEUS, contrast-enhanced ultrasound; MWSA, microwave ablation.

\section{Statistical analysis}

The quantitative data were expressed as mean \pm standard deviation. Continuous variables and categorical variables were compared using student's $t$ test and chi-square test, respectively. Cumulative overall survival (OS) rate was estimated in months using the Kaplan-Meier method, from the beginning of the ablation to death or the last visit, and the differences between subgroups of patients in univariate analysis were determined using the log-rank test. Eleven possible factors affecting survival were analyzed after their transformation in dichotomous variables (i.e. age, sex, presence of cirrhosis, total bilirubin level, Ca 19-9 value, alpha-fetoprotein value, number of nodules, diameter of nodules, surgical recurrence, primary treatment, and type of treatment). Variables with a $p$ value $\leq 0.10$ on univariate analysis were included in multivariate regression analysis by using Cox's stepwise regression. Statistical significance was defined by a $p$ value $<0.05$ in a two-tailed test. Statistical analysis was performed using the SPSS 22.0 statistical software (SPSS Inc., Chicago, IL, USA).

\section{Results}

The clinical, laboratory and radiological characteristics were comparable between the two groups $(p>0.05)$. The follow-up

Table 3. The enhancement pattern of the intrahepatic cholangiocarcinoma treated with RFA during the arterial phase on CEUS and CECT/CEMRI, and according to lesion size

\begin{tabular}{|c|c|c|c|c|c|}
\hline \multirow[b]{2}{*}{ CECT or CEMRI } & \multicolumn{5}{|l|}{ CEUS (\%) } \\
\hline & Type I & Type II & Type III & Type IV & Total \\
\hline Type I & $27(56.2)$ & $4(8.3)$ & $0(0)$ & $0(0)$ & $31(64.5)$ \\
\hline Type II & $0(0)$ & $9(18.7)$ & $0(0)$ & $2(4.2)$ & $11(22.9)$ \\
\hline Type III & $2(4.2)$ & $0(0)$ & $4(8.3)$ & $0(0)$ & $6(12.5)$ \\
\hline Type IV & $0(0)$ & $0(0)$ & $0(0)$ & $0(0)$ & $0(0)$ \\
\hline Total & $29(60.4)$ & $13(27.0)$ & $4(8.3)$ & $2(4.2)$ & 48 \\
\hline \multicolumn{6}{|c|}{ Size, median (range): $3.1 \mathrm{~cm}(2-8 \mathrm{~cm})$} \\
\hline$\leq 3.0 \mathrm{~cm}$ & $4(8.3)$ & $0(0)$ & $4(8.3)$ & $0(0)$ & $8(16.7)$ \\
\hline $3.1-5.0 \mathrm{~cm}$ & $22(45.8)$ & $10(20,8)$ & $0(0)$ & $0(0)$ & $32(66.7)$ \\
\hline$>5 \mathrm{~cm}$ & $3(6.2)$ & $3(6.2)$ & $0(0)$ & $2(4.2)$ & $8(16.7)$ \\
\hline Total & $29(60.4)$ & $13(27.0)$ & $4(8.3)$ & $2(4.2)$ & 48 \\
\hline
\end{tabular}

Type I (peripheral irregular rim-like hyperenhancement);Type II (diffuse heterogeneous hyperenhancement); Type III, diffuse homogeneous hyperenhancement); Type IV (diffuse heterogeneous hypoenhancement).

Abbreviations: CEMRI, contrast-enhanced magnetic resonance imaging; CETC, contrast-enhanced computed tomography; CEUS, contrast-enhanced ultrasound; RFA, radiofrequency ablation. 


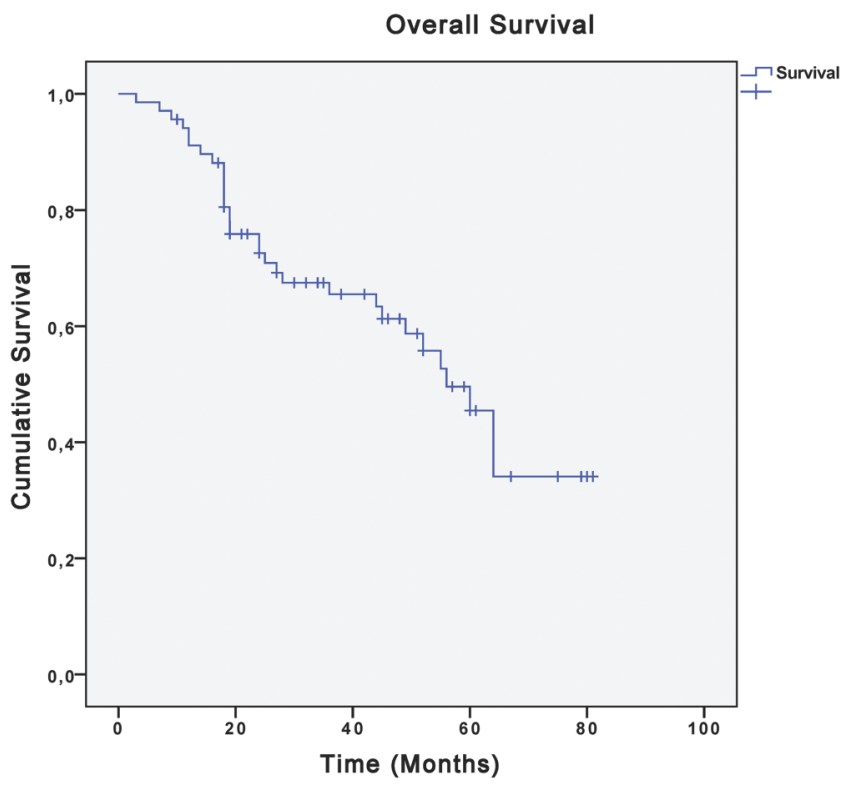

Fig. 1. Overall Survival of all patients.

of the study ranged between 8 and 86 months (median: 48). OS of all patients at $12,36,60$ and 80 months was $88 \%, 65 \%$, $45 \%$ and $34 \%$, respectively (Fig. 1). Patients treated with MWSA survived longer than patients treated with RFA (OS was $95 \%, 75 \%, 68 \%$ and $68 \%$ at $12,36,60$ and 80 months, respectively, in the MWSA group and $86 \%, 53 \%$, $26 \%$ and $13 \%$, respectively, in the RFA group $(p<0.005)$ (Fig. 2). OS of patients with nodules $\leq 3 \mathrm{~cm}$ was better in the MWSA group than in the RFA group (93\%, 67\%, 60\%

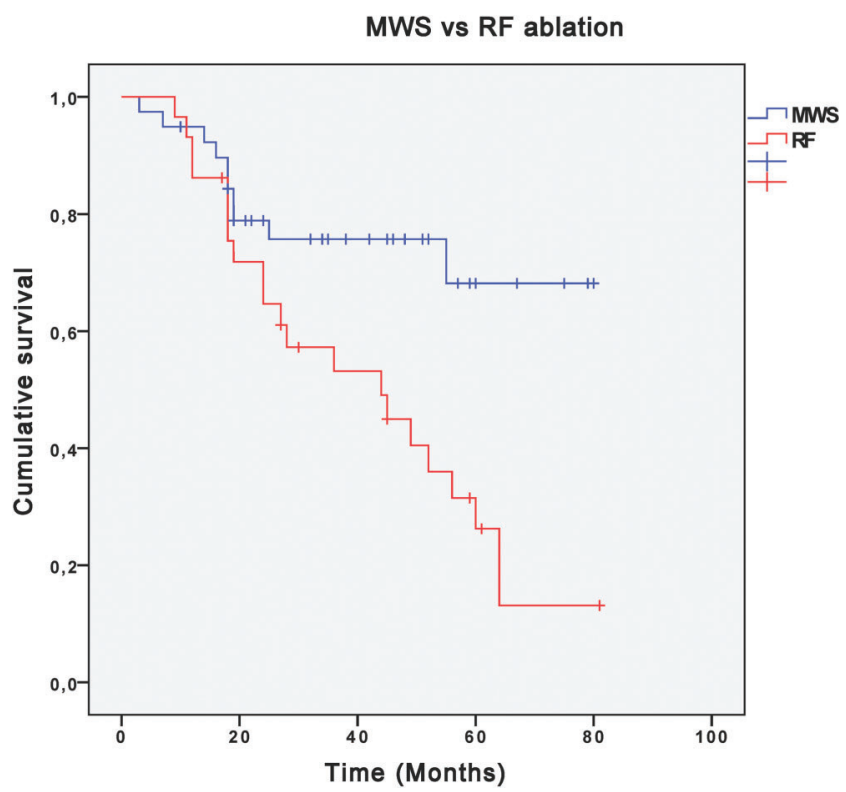

Fig. 2. Overall survival of patients treated with MWSA vs. patients treated with RFA.

Abbreviations: MWSA, microwave ablation; RFA, radiofrequency ablation.

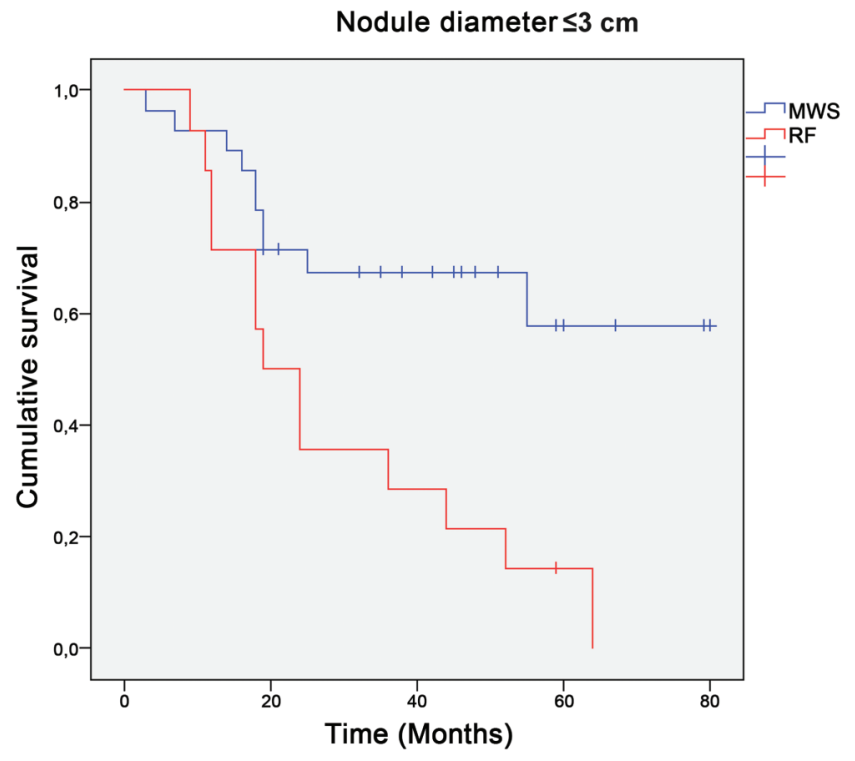

Fig. 3. Overall survival of patients with nodules $\leq 3 \mathrm{~cm}$ treated with MWSA vs. patients treated with RFA.

Abbreviations: MWSA, microwave ablation; RFA, radiofrequency ablation.

and $58 \%$ at $12,36,60$ and 80 months, respectively, vs. $86 \%, 29 \%, 14$ and $0 \%, p<0.005$ ) (Fig. 3). In the subgroup of patients with ICC nodules $\leq 4 \mathrm{~cm}$, OS of patients treated with MWSA was better than those treated with RFA $(p<0.0005)$ (Fig. 4). This statistically significant difference was no longer evident when diameter of nodules was $>4 \mathrm{~cm}(p=0.25)$.

Disease-free survival was better in the MWSA-treated group, with respect to the RFA-treated group $(p<0.005)$

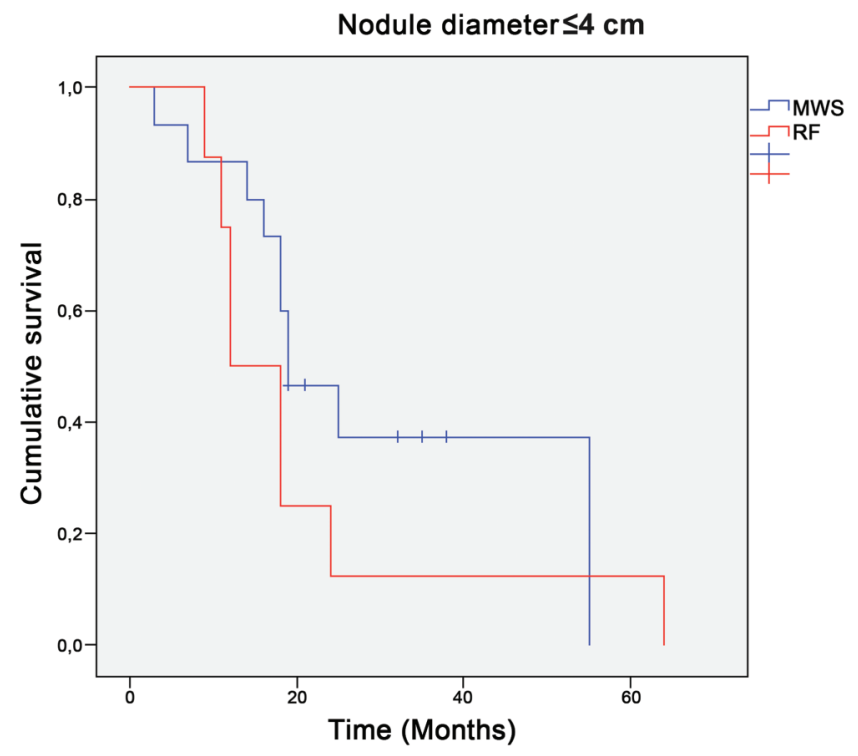

Fig. 4. Overall survival of patients with nodules $\leq 4 \mathrm{~cm}$ treated with MWSA vs. patients treated with RFA.

Abbreviations: MWSA, microwave ablation; RFA, radiofrequency ablation. 
Giorgio A. et al: Ablation of intrahepatic cholangiocarcinoma

Table 4. Significant variables for overall survival in the univariate and multivariate analysis

\begin{tabular}{|c|c|c|c|c|}
\hline Significant variables & $\begin{array}{l}\text { Univariate analysis, } \\
\text { OR }(95 \% \mathrm{CI})\end{array}$ & $p$ & $\begin{array}{l}\text { Multivariate analysis, } \\
\text { OR }(95 \% \mathrm{CI})\end{array}$ & $p$ \\
\hline Age in years: $\leq 60$ vs. $>60$ & $1.122(0.738-1.727)$ & & & \\
\hline Male sex: yes/no & $0.929(0.532-1.1791)$ & & & \\
\hline Cirrhosis: yes/no & $0.989(0.841-2.788)$ & 0.002 & & \\
\hline Total bilirubin level in $\mathrm{mg} / \mathrm{dL}: \leq 2.5$ vs. $>2.5$ & $3.020(1.631-5.579)$ & & & \\
\hline Ca 19.9 in IU/mL: $\leq 40$ vs. $>40$ & $1.149(0.748-1.729)$ & & & \\
\hline AFP in $\mathrm{mg} / \mathrm{dL}: \leq 20$ vs. $>20$ & $1.151(0.751-1.1734)$ & & & \\
\hline Size of nodules in $\mathrm{cm}: \leq 3$ vs. $>3$ & $1.161(0.750-1.359)$ & 0.003 & $1.527(1.868-2.077)$ & 0.002 \\
\hline Number of nodules 1 vs. $>1$ & $1.37(0.791-1.733)$ & 0.003 & & \\
\hline Primary treatment vs. surgical recurrence & $0.989(0.754-1.311)$ & 0.986 & & \\
\hline MWSA vs. RFA & $2.079(1.191-2.730)$ & 0.002 & $1.899(1.227-2.888)$ & 0.001 \\
\hline
\end{tabular}

Abbreviations: AFP, alpha-fetoprotein; CI, confidence interval; MWSA, microwave ablation; OR, odds ratio; RFA, radiofrequency ablation.

(Supplementary Fig. 1). Progression-free survival was $79 \%$, $59 \%, 55 \%$ and $55 \%$ at $12,36,60$ and 80 months, respectively, in the MWSA group and $69 \%, 51 \%, 8.5 \%$ and $8.5 \%$ at $12,36,60$ and 80 months, respectively, in the RFA group, with a significant statistical difference $(p<0.005)$ (Supplementary Fig. 2). On univariate analysis, the presence of cirrhosis, diameter of nodules, size of nodules, and type of treatment were independent factors affecting survival. On multivariate analysis, only diameter of nodules and type of treatment were independent factors predicting survival (Table 4). No major complications were observed after procedures and the hospital stay ranged between 1 and 4 days (mean 1.7).

\section{Discussion}

Our retrospective study showed that MWSA is superior to RFA in treatment of patients with ICC not amenable to surgical resection. Furthermore, results of our series show that MWSA achieves a better survival in patients with nodules $\leq 3 \mathrm{~cm}$ as well as those up to $4 \mathrm{~cm}$, as compared to RFA. In our opinion, this can be explained considering the intrinsic characteristics of MWSA with respect to RFA. In fact, compared to RFA, MWSA induces a larger volume of necrosis in a faster time and determinates a spherical shape of the ablated area. ${ }^{13,14}$ Therefore, from an interventional point of view, it is conceivable that MWSA, inducing a better local tumor control, allows an increased survival, both considering the results in terms of OS and considering the disease-free survival and progressionfree survival rates of our series.

To our knowledge, there are no studies comparing the two thermal techniques in treating ICC not amenable for surgical resection. Generally, previous studies have reported only results using one of the two techniques in treating ICC patients. Recently, however, Takahashi et al. ${ }^{17}$ retrospectively reviewed the outcome of 20 patients with 50 ICC nodules (mean size: $1.8 \pm 1.3 \mathrm{~cm}$ ) treated mainly with RFA $(88 \%$ of nodules) or MWSA between 2006 and 2015. In their experience, there was no difference between the two techniques for local tumor progression. We must consider that in the study of Takahashi and colleagues, ${ }^{17}$ the ICC-treated nodules were too small and the difference between the two groups (RFA and MWSA) was clearly unbalanced in favor of the RFA ( $88 \%$ of nodules).

Zhang et al. ${ }^{18}$ reported the clinical and survival outcomes of 101 ICC patients treated with MWSA between 2009 and 2016. According to their results, Child-Pugh class of cirrhosis and the number of nodules were independent factors affecting survival. Xu et al. ${ }^{19}$ reported a retrospective comparison between MWSA and surgical resection in patients with recurrent ICC. They concluded that percutaneous MWSA can be considered comparable with surgical resection and should be a valid alternative to surgery in treating recurrent ICC after hepatectomy. Our results, although retrospective, seem to show that MWSA could be a valid alternative to surgery not only in patients with nodules $\leq 3 \mathrm{~cm}$ but also in the subgroup of ICC patients with nodules between 3 and $4 \mathrm{~cm}$ in diameter.

Our study has many limitations. First, it is a retrospective study, with all the implications that this has in the analysis of the results. In fact, it is well known that retrospective studies give less reliable results compared to prospective studies. Moreover, in our study the indication of procedure (i.e. RFA or MWSA) was not chosen in a randomized manner but on a case-by-case basis depending on the patient and the operator. Second, the type of ablation devices used in our series was quite different among the five different Interventional Units and this could have affected the results. It should also be considered that, at the beginning of the study, the skills of the physicians involved in the ablation procedures could have been not exactly the same. Finally, only a single electrode needle (in the case of RFA) and only a single antenna (in the case of MWSA) were used in all procedures. Probably, the use of cluster RF electrode needles or more than one MWS antenna could have induced a better local tumor control and a better survival in cases of larger nodules. On the other hand, it is conceivable that when using multiple applicators, the risk of bleeding would be doubled or tripled while, in our series, no bleeding event was observed.

In conclusion, in our experience, MWSA seems to be superior to RFA in treatment of ICC nodules in patients unfit for surgical resection, in nodules $\leq 3 \mathrm{~cm}$ as well as in nodules up to $4 \mathrm{~cm}$, inducing better $\mathrm{OS}$ and better local tumor control. Because our results were obtained from a real-life retrospective study and from a limited number of centers, 
further prospective controlled randomized trials with a larger number of patients are needed to confirm our results.

\section{Acknowledgments}

The authors thank Dr. Massimo De Luca, M.D., Liver Unit at Cardarelli Hospital, Naples, Italy, for his assistance in the preparation of the manuscript.

\section{Conflict of interest}

The authors have no conflict of interests related to this publication.

\section{Author contributions}

Study concept and design (AG), acquisition of data (AG, PG, LM), analysis and interpretation of data (AG, PG, LM), drafting of the manuscript (AG,VG), critical revision of the manuscript for important intellectual content, and administrative, technical, or material support ( $A G, P G, L M, B S, A D, N C, C C, F S$, FDB, EC, SS, VG), study supervision (AG, PG,VG).

\section{References}

[1] Gupta A, Dixon E. Epidemiology and risk factors: intrahepatic cholangiocarcinoma. Hepatobiliary Surg Nutr 2017;6:101-104. doi: 10.21037/hbsn. 2017.01.02.

[2] Bertuccio $P$, Malvezzi M, Carioli G, Hashim D, Boffetta $P, E l-S e r a g ~ H B$, et al. Global trends in mortality from intrahepatic and extrahepatic cholangiocarcinoma. J Hepatol 2019;71:104-114. doi: 10.1016/j.jhep.2019.03.013.

[3] Weber SM, Ribero D, O'Reilly EM, Kokudo N, Miyazaki M, Pawlik TM. Intrahepatic cholangiocarcinoma: expert consensus statement. HPB (Oxford) 2015;17:669-680. doi: 10.1111/hpb.12441.

[4] Carrafiello G, Laganà D, Cotta E, Mangini M, Fontana F, Bandiera F, et al. Radiofrequency ablation of intrahepatic cholangiocarcinoma: preliminary experience. Cardiovasc Intervent Radiol 2010;33:835-839. doi: 10 1007/s00270-010-9849-3

[5] Chiou YY, Hwang JI, Chou YH, Wang HK, Chiang JH, Chang CY. Percutaneous ultrasound-guided radiofrequency ablation of intrahepatic cholangiocarcinoma. Kaohsiung J Med Sci 2005;21:304-309. doi: 10.1016/S1607-551X (09)70125-1.
[6] Fu Y, Yang W, Wu W, Yan K, Xing BC, Chen MH. Radiofrequency ablation in the management of unresectable intrahepatic cholangiocarcinoma. J Vasc Interv Radiol 2012;23:642-649. doi: 10.1016/j.jvir.2012.01.081.

[7] Giorgio A, Calisti G, DE Stefano G, Farella N, DI Sarno A, Amendola F, et al. Radiofrequency ablation for intrahepatic cholangiocarcinoma: retrospective analysis of a single centre experience. Anticancer Res 2011;31: 4575-4580.

[8] Kim JH, Won HJ, Shin YM, Kim KA, Kim PN. Radiofrequency ablation for the treatment of primary intrahepatic cholangiocarcinoma. AJR Am J Roentgenol 2011;196:W205-W209. doi: 10.2214/AJR.10.4937.

[9] Xu HX, Wang Y, Lu MD, Liu LN. Percutaneous ultrasound-guided thermal ablation for intrahepatic cholangiocarcinoma. Br J Radiol 2012;85:10781084. doi: $10.1259 / \mathrm{bjr} / 24563774$

[10] Mizrahi JD, Abdel-Wahab R, Javle M. Clinical trials and novel/emerging treatment. In: Pawlik T, Cloyd J, Dillhoff, editors. Intrahepatic Cholangiocarcinoma. Springer, Cham; 2019:183-208. doi: 10.1007/978-3-030-222581 13.

[11] Shindoh J. Ablative therapies for intrahepatic cholangiocarcinoma. Hepatobiliary Surg Nutr 2017;6:2-6. doi: 10.21037/hbsn.2016.09.07.

[12] Giorgio A, Gatti P, Matteucci P, Giorgio V. Ablative therapies for intrahepatic cholangiocarcinoma. Hepatobiliary Surg Nutr 2018;7:192-194. doi: 10. 21037/hbsn.2018.02.06.

[13] Giorgio A, Gatti P, Montesarchio L, Merola MG, Amendola F, Calvanese A, et al. Microwave ablation in intermediate hepatocellular carcinoma in cirrhosis: An Italian multicenter prospective study. J Clin Transl Hepatol 2018;6: 251-257. doi: 10.14218/JCTH.2018.00013.

[14] Poggi G, Montagna B, DI Cesare P, Riva G, Bernardo G, Mazzucco M, et al. Microwave ablation of hepatocellular carcinoma using a new percutaneous device: preliminary results. Anticancer Res 2013;33:1221-1227.

[15] Chen LD, Xu HX, Xie XY, Lu MD, Xu ZF, Liu GJ, et al. Enhancement patterns of intrahepatic cholangiocarcinoma: comparison between contrast-enhanced ultrasound and contrast-enhanced CT. Br J Radiol 2008;81:881-889. doi: $10.1259 / \mathrm{bjr} / 22318475$.

[16] Giorgio A, Merola MG, Montesarchio L, Merola F, Gatti P, Coppola C, et al. Percutaneous radiofrequency ablation of hepatocellular carcinoma in cirrhosis: analysis of complications in a single centre over 20 years. Br J Radiol 2017;90: 20160804. doi: 10.1259/bjr.20160804.

[17] Takahashi EA, Kinsman KA, Schmit GD, Atwell TD, Schmitz J], Welch BT, et al. Thermal ablation of intrahepatic cholangiocarcinoma: Safety, efficacy, and factors affecting local tumor progression. Abdom Radiol (NY) 2018;43: 3487-3492. doi: 10.1007/s00261-018-1656-3.

[18] Zhang K, Yu J, Yu X, Han Z, Cheng Z, Liu F, et al. Clinical and survival outcomes of percutaneous microwave ablation for intrahepatic cholangiocarcinoma. Int J Hyperthermia 2018;34:292-297. doi: 10.1080/02656736. 2017.1327678

[19] Xu C, Li L, Xu W, Du C, Yang L, Tong J, et al. Ultrasound-guided percutaneous microwave ablation versus surgical resection for recurrent intrahepatic cholangiocarcinoma: intermediate-term results. Int J Hyperthermia 2019;36: 351-358. doi: 10.1080/02656736.2019.1571247. 